\title{
Duloxetine for Al-associated joint pain in breast cancer patients
}

\author{
Moe Tsuda, Masakazu Toi \\ Department of Breast Surgery, Kyoto University Graduate School of Medicine, Kyoto, Japan \\ Correspondence to: Prof. Masakazu Toi. Department of Breast Surgery, Kyoto University Graduate School of Medicine, 54 Shogoin-kawaharacho, \\ Sakyo-ku, Kyoto 606-8507, Japan. Email: toi@kuhp.kyoto-u.ac.jp. \\ Comment on: Henry NL, Unger JM, Schott AF, et al. Randomized, multicenter, placebo-controlled clinical trial of duloxetine versus placebo for \\ aromatase inhibitor-associated arthralgias in early-stage breast cancer: SWOG S1202. J Clin Oncol 2018;36:326-32.
}

Submitted Feb 15, 2018. Accepted for publication Mar 09, 2018.

doi: $10.21037 /$ tcr.2018.03.28

View this article at: http://dx.doi.org/10.21037/tcr.2018.03.28

Aromatase inhibitors (AIs) are the standard of care for treatment of both early and advanced hormone-sensitive breast cancer in postmenopausal women. Although AIs have been considered to be well-tolerated, only $50 \%$ of patients can complete the full course of the treatment, at the optimal schedule (1). A prospective randomized trial revealed that $32 \%$ of patients discontinued initial AI therapy within 2 years of initiation, because of adverse events, and $75 \%$ of them did so due to AI-associated musculoskeletal symptoms (AIMSS), including joint pain and stiffness (2). AIMSS reportedly occurs in up to $50 \%$ of breast cancer patients treated with AIs $(3,4)$. Therefore, effective management of AIMSS is necessary for continuing AIs without compromising the quality of life (QOL). However, a standard of care for AIMSS, especially in terms of pharmacological interventions, is yet to be validated.

Duloxetine, a selective serotonin and norepinephrine reuptake inhibitor (SNRI), is thought to enhance descending pain inhibitory pathways by inhibiting the reuptake of serotonin and norepinephrine. Clinical trials have revealed that duloxetine decreases chronic pain related to various etiologies including diabetic neuropathy, fibromyalgia, and osteoarthritis, along with chronic low back pain (5).

Henry et al., conducted a randomized, multi-center, placebo-controlled clinical trial, to assess the efficacy of duloxetine in relieving pain related to AIMSS in early breast cancer patients (6). A total of 299 early-stage breast cancer patients with an average joint pain score of $\geq 4$ out of 10 in whom pain developed or worsened since AI therapy initiation, were randomly assigned to duloxetine or placebo for 12 weeks (1:1), which was then tapered off over a period of 1 week. Patients who had been taking AIs for a period of 21 days to 3 years were included in the study. Joint pain, pain interference, functioning, QOL, and depression were assessed at weeks 2, 6, and 12 (during intervention), as well as at week 24 (12 weeks after discontinuing the full-dose intervention). Patients were stratified by baseline pain score assessed by the Brief Pain Inventory (BPI), and prior taxane use. The duration of AI use was within 1 year in $64 \%$ of the patients. Results showed that the average pain score through 12 weeks, which was the primary endpoint, was reduced to a slightly greater extent (by 0.82 points) in the duloxetine arm than in the placebo arm (95\% CI, -1.24 to $-0.40, \mathrm{P}=0.0002)$. Importantly, $52 \%$ of the patients treated with duloxetine immediately achieved clinically meaningful improvement in pain (defined as a $\geq 2$-point decrease from baseline), within 2 weeks after initiation of the therapy. At week 6, this increased to $68 \%$ and was sustained until discontinuation of duloxetine. Although adverse events were seen more frequently in the duloxetine arm, most were of grade 1-2 severity and included fatigue, nausea, dry mouth, and headache.

Methodological assessment of pain related to AIMSS was the strength of this study. AIMSS are often underreported by physicians in clinical practice, as they are highly subjective and difficult to be recognized, classified, and graded systematically. In this trial, patientreported outcomes were simultaneously assessed by four questionnaire tools. As a result, reliable and consistent improvement with duloxetine in terms of average pain, worst pain, stiffness, pain interference, and functioning was noted.

In this trial, more than half of patients in the duloxetine 
arm reported clinically meaningful ( $\geq 2$ points) improvement in pain. However, the benefit noted with duloxetine over placebo was only 0.82 points, which may not be enough to suggest a dramatic change in clinical practice. Several factors including natural history of AIMSS without intervention, patient selection, active ingredients within the placebo itself, and "placebo effect" caused by patients' expectations for interventions, may probably have a role in this (7).

The "placebo effect" has been reported to have a significant role in studies related to symptom management. Previous reports revealed that the magnitude of placebo effect was as high as $30-50 \%$ in trials investigating pharmacological management of cancer treatmentinduced toxicities such as vasomotor symptoms, fatigue, and neuropathy (7). In a trial comparing omega-3-fatty acids (O3-FAs) versus placebo for 24 weeks in patients with AIMSS, no significant difference in pain improvement was noted between the two arms $(61 \%$ and $57 \%$ of patients reported improvement of pain, in O3-FAs, and placebo arms, respectively), despite significant improvement in the O3-FAs arm compared to baseline (8). Contrarily, two randomized trials evaluating non-pharmacological management for AIMSS reported significant improvement following such interventions, without any placebo effect. One was a trial comparing true acupuncture with sham acupuncture, which revealed $50 \%$ decrease in pain with true acupuncture, whereas there was no change in pain intensity with sham acupuncture (9), and the other trial comparing exercise intervention with usual care reported $29 \%$ decrease in pain score following exercise, whereas there was no change in pain intensity with usual care (10). Importantly, Henry et al. demonstrated superiority of duloxetine to placebo, regardless of significant improvement in the placebo arm (6). Furthermore, $71 \%$ of patients in the duloxetine arm reported that the intervention was beneficial, while only $49 \%$ in the placebo arm noted beneficial effects. Hence, it can be suggested that duloxetine is effective in the management of AIMSS.

However, the efficacy of placebo cannot be ignored. While the magnitude of "placebo effect" would be lesser in clinical practice (compared to clinical trials), its advantages in terms of biochemical safety and reduced cost (compared with the actual drug), may need to be considered. Placebo as an option for treatment of AIMSS as well as the ethical implications will need further research. A recent randomized controlled trial related to irritable bowel syndrome (IBS) revealed that placebo worked effectively even without blinding (11). Patients were randomized to either openlabel placebo pills (presented as "placebo pills made of an inert substance, like sugar pills, that have been shown in clinical studies to produce significant improvement in IBS symptoms through mind-body self-healing processes") or no-treatment (control group), with the same quality of interaction with providers. A significant improvement in IBS symptoms and a better QOL were noted in the open label placebo group compared to the control group. Similar results were also reported in a small pilot study evaluating antidepressant drugs in patients with depression (12). Several national studies revealed that most physicians regularly prescribe placebo, and consider it ethical $(13,14)$. However, further trials are needed to confirm the possibility of placebo as a treatment option, especially in view of longterm efficacy and toxicity.

Epidemiologically, nearly $50 \%$ of healthy postmenopausal women develop joint and muscle pain without AIs (15). However, no specific biomarker or symptom classification has been established to distinguish AIMSS from other etiologies of musculoskeletal pain. The pathophysiology of AIMSS is also not clear. It has been hypothesized that estrogen deprivation caused by AIs results in musculoskeletal pain through numerous mechanisms. Preliminary evidences have demonstrated anti-nociceptive and anti-inflammatory effects of estrogens. Additionally, the protective effect of estrogens on structural integrity of articular cartilage has been suggested (15). Dizdar et al. reported increased tendon thickness and higher rates of effusion in hand joints/tendons on musculoskeletal sonography in women with AI-induced arthralgia compared to those who never received AIs (16). Although several factors such as dehydroepiandrosterone sulfate (DHEAS), insulin-like growth factor-I (IGF-I), interleukin-17 (IL-17), and vitamin $\mathrm{D}$, have been suggested to be associated with the development of AIMSS, the underlying mechanisms are not known $(15,17)$.

Assessment of the relationship between AIMSS and survival outcomes also revealed mixed results. In retrospective exploratory analyses of the Tamoxifen Exemestane Adjuvant Multinational (TEAM), Arimidex, Tamoxifen, Alone or in Combination Trial (ATAC), and Breast International Group (BIG) 1-98 trial, musculoskeletal symptoms were suggested to be associated with better survival outcomes (18-20). In contrast, the MA.27 study revealed no association between these aspects (21). Interestingly, the incidence of AIMSS was higher in ATAC than in MA.27. However, an adherence rate of $88 \%$ among 
patients with symptoms, and $84 \%$ among those without symptoms was noted in ATAC patients, whereas it was only $70 \%$ in MA.27 patients; $31.6 \%$ of all patients in the MA. 27 study discontinued AIs mainly due to AIMSS (21). These results suggest that AIMSS can potentially be a prognostic factor of AIs when associated with good adherence. Therefore, management of AIMSS is essential to ensure long term adherence to life-saving AIs.

A new step in the management of AIMSS is suggested. Duloxetine can potentially be a new standard of care for patients with AIMSS. Further understanding of etiology would be helpful to improve the management of AIMSS and increase adherence to AIs.

\section{Acknowledgments}

Funding: None.

\section{Footnote}

Provenance and Peer Review: This article was commissioned and reviewed by the Section Editor Tao Qin (Department of Medical Oncology, Sun Yat-sen Memorial Hospital, Sun Yat-sen University, Guangzhou, China).

Conflicts of Interest: Dr. Moe Tsuda has no conflicts of interest to declare. Dr. Masakazu Toi received honoraria from Eli Lilly and Company, and received grant/research support form Eli Lilly and Company for oncological studies.

Ethical Statement: The authors are accountable for all aspects of the work in ensuring that questions related to the accuracy or integrity of any part of the work are appropriately investigated and resolved.

Open Access Statement: This is an Open Access article distributed in accordance with the Creative Commons Attribution-NonCommercial-NoDerivs 4.0 International License (CC BY-NC-ND 4.0), which permits the noncommercial replication and distribution of the article with the strict proviso that no changes or edits are made and the original work is properly cited (including links to both the formal publication through the relevant DOI and the license). See: https://creativecommons.org/licenses/by-nc-nd/4.0/.

\section{References}

1. Hershman DL, Kushi LH, Shao T, et al. Early discontinuation and nonadherence to adjuvant hormonal therapy in a cohort of 8,769 early-stage breast cancer patients. J Clin Oncol 2010;28:4120-8.

2. Henry NL, Azzouz F, Desta Z, et al. Predictors of aromatase inhibitor discontinuation as a result of treatment-emergent symptoms in early-stage breast cancer. J Clin Oncol 2012;30:936-42.

3. Hershman DL, Shao T, Kushi LH, et al. Early discontinuation and non-adherence to adjuvant hormonal therapy are associated with increased mortality in women with breast cancer. Breast Cancer Res Treat 2011;126:529-37.

4. Hadji P, Jackisch C, Bolten W, et al. COMPliance and Arthralgia in Clinical Therapy: the COMPACT trial, assessing the incidence of arthralgia, and compliance within the first year of adjuvant anastrozole therapy. Ann Oncol 2014;25:372-7.

5. Skljarevski V, Zhang S, Desaiah D, et al. Duloxetine versus placebo in patients with chronic low back pain: a 12week, fixed-dose, randomized, double-blind trial. J Pain 2010;11:1282-90.

6. Henry NL, Unger JM, Schott AF, et al. Randomized, multicenter, placebo-controlled clinical trial of duloxetine versus placebo for aromatase inhibitor-associated arthralgias in early-stage breast cancer: SWOG S1202. J Clin Oncol 2018;36:326-32.

7. Henry NL, Griggs JJ. The Power of the Placebo in Symptom Management. J Clin Oncol 2015;33:1870-2.

8. Hershman DL, Unger JM, Crew KD, et al. Randomized Multicenter Placebo-Controlled Trial of Omega-3 Fatty Acids for the Control of Aromatase Inhibitor-Induced Musculoskeletal Pain: SWOG S0927. J Clin Oncol 2015;33:1910-7.

9. Crew KD, Capodice JL, Greenlee H, et al. Randomized, blinded, sham-controlled trial of acupuncture for the management of aromatase inhibitor-associated joint symptoms in women with early-stage breast cancer. J Clin Oncol 2010;28:1154-60.

10. Irwin ML, Cartmel B, Gross CP, et al. Randomized exercise trial of aromatase inhibitor-induced arthralgia in breast cancer survivors. J Clin Oncol 2015;33:1104-11.

11. Kaptchuk TJ, Friedlander E, Kelley JM, et al. Placebos without deception: a randomized controlled trial in irritable bowel syndrome. PloS One 2010;5:e15591.

12. Kelley JM, Kaptchuk TJ, Cusin C, et al. Open-label placebo for major depressive disorder: a pilot randomized controlled trial. Psychother Psychosom 2012;81:312-4.

13. Tilburt JC, Emanuel EJ, Kaptchuk TJ, et al. Prescribing 
"placebo treatments": results of national survey of US internists and rheumatologists. BMJ 2008;337:a1938.

14. Howick J, Bishop FL, Heneghan C, et al. Placebo use in the United Kingdom: results from a national survey of primary care practitioners. PLoS One 2013;8:e58247.

15. Din OS, Dodwell D, Wakefield RJ, et al. Aromatase inhibitor-induced arthralgia in early breast cancer: what do we know and how can we find out more? Breast Cancer Res Treat 2010;120:525-38.

16. Dizdar O, Ozcakar L, Malas FU, et al. Sonographic and electrodiagnostic evaluations in patients with aromatase inhibitor-related arthralgia. J Clin Oncol 2009;27:4955-60.

17. Oestergaard S, Sondergaard BC, Hoegh-Andersen P, et al. Effects of ovariectomy and estrogen therapy on type II collagen degradation and structural integrity of articular cartilage in rats: implications of the time of initiation. Arthritis Rheum 2006;54:2441-51.

Cite this article as: Tsuda M, Toi M. Duloxetine for AIassociated joint pain in breast cancer patients. Transl Cancer Res 2018; 7(Suppl 4):S480-S483. doi: 10.21037/tcr.2018.03.28
18. Fontein DB, Seynaeve C, Hadji P, et al. Specific adverse events predict survival benefit in patients treated with tamoxifen or aromatase inhibitors: an international tamoxifen exemestane adjuvant multinational trial analysis. J Clin Oncol 2013;31:2257-64.

19. Cuzick J, Sestak I, Cella D, et al. Treatment-emergent endocrine symptoms and the risk of breast cancer recurrence: a retrospective analysis of the ATAC trial. Lancet Oncol 2008;9:1143-8.

20. Huober J, Cole BF, Rabaglio M, et al. Symptoms of endocrine treatment and outcome in the BIG 1-98 study. Breast Cancer Res Treat 2014;143:159-69.

21. Stearns V, Chapman JA, Ma CX, et al. Treatment-associated musculoskeletal and vasomotor symptoms and relapse-free survival in the NCIC CTG MA.27 adjuvant breast cancer aromatase inhibitor trial. J Clin Oncol 2015;33:265-71. 